\title{
OXYURIASIS EN LA INFANCIA
}

\author{
Ensayo terapéutico con citrato y fosfato de dietilendiamina (Piperazina)
}

\author{
DrS. FERNANDO DONOOSO e ISIDORO COLODRO
}

Cátedra de Parasitologia del Prol. Amador Neghme. Universidad de Chíle.

\section{INTRODUCCIÓN *}

El Enterobius vermicularis, oxyuro o vulgarmente "pidulle", es una de las enteroparasitosis más difundida en todo el

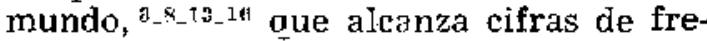
cuencia superiores al $40 \%$ en la población entre 6 a 15 años de edad en nuestro país. Por otra varte, las características biológica $\mathrm{y}$ epidemiológicas de este parásito ${ }^{3-8}{ }_{-} 15$, contribuyen al fracaso de muchos de Ios tratamien.os realizados, ya sea en individuos aislados o en grupos familiares. Desde la introdución en Parasitología de la droga llamada dietilendiamina (Piperazina) ${ }^{11}{ }_{1}^{12}$ y su empleo posterior en el iratamiento de las enterobiásis ${ }^{2}{ }^{12}{ }_{-} 16$, contamos con un valioso agente terapéutico oue cumple en gran parte las condiciones de droga ideal para el tratamiento de esta enteroparasitosis. Es así como la experiencia mundial revela la eficacia de diversas sales de la piperazina: hexahidrato, citrato, adipato, en la terapia de oxyuriasis, con resultados variables de acuerdo a las dosis $\mathrm{y}$ esquemas terapéuticos utilizados ${ }^{2}{ }_{3}+12{ }^{2}+$. En la actualidad existe cierta tendencia a reducir la duración de los tratamienios $\mathrm{y}$ algunos autores han ensayado esquemas terapéuticos de sólo 2. 3 ó 4 días de duración ${ }^{1}$, , pero los resultados obtenidos son muy disnares debidos, al parecer, a diferencias en el medio ambiente de trabajo y en la técnica del control parasitológico. Recientemente hemos podido contar con una nueva sal de piperazina, el fosfato, y hemos creído necesario conocer sus propiedades curativas ensayándola en el tratamiento de pacientes infectados por Enterobius vermicularis, al mismo tiempo que comparar su acción, con el citrato de piperazina, derivado de reconocida eficacia. Por tratarse de un ensayo terapéutico comparativo fú́ necesario guiarse por los escuemas de tratamiento clásicos en esta materia.

\section{MATERIAL Y MÉTODO}

Nuestro trabajo consistió en el tratamiento de 50 pacientes comprobadamente oxyurióticos gue concurrieron a la Policlínica de Enffermedades Parasitarias, de la Cátedra de Parasitología de la Universidad de Chile, divididos en dos grupos, según la droga utilizada. El Grupo I estuvo formado por 23 niños de ambos sexos, cuyas edades fluctuaron entre 1 y 11 años y que recibieron tratamiento con jarabe de Citra dosis de 80 milígramos por kilógramo de peso y por día, administrado durante 15 días consecutivos. El Grupo II comprendió a 27 niños, de ambos sexos, que fueron sometidos al mismo esquema terapéutico, pero con tabletas dé Fosfato de piperazina. El jarabe de Citrato de piperazina (Antepar) contiene el equivalente de 100 milígramos de hexahidrato por cada mililitro y las iabletas de Fosfato de piperazina (Exelmín), vienen dosificadas a 250 milígramos cada una. En ambos grupos se utilizó una dosis diaria máxima de 3 gramos para los niños. Ambos, el Citrato y el Fosfato de piperazina, fueron administrados en una dosis única diaria, en ayunas, aconsejándose el fraccionamiento o dilución de las tabletas en los niños menores.

Tanto el diagnóstico como los controles realizados con posterioridad al tratamiento se hicieron medianie la técnica de la cinta de papel celofán engomada introducida por Graham ${ }^{10}$. El control en el Grupo I consistió en el estudio de dos series de cinco placas de papel celofán cada una, tomadas en días consecutivos a $\operatorname{los} 7$ y 14 días después de finalizada la cura; en algunos niños de este grupo pudo realizarse un tercer control en igual forma, entre 2 y 6 meses después del tratamiento. El Grup̣o II fué controlado mediante dos series de 10 placas de papel celofán engomado, tomadas también 
en días sucesivos, a los 3 y 14 días después de la cura.

La onganización del trabajo en nuestra Policlínica nos permitió siempre tratar $\checkmark$ controlar a grupos familiares completos: de este modo los componentes de una familia que se hallaban libres de oxyuros pudieron ser tratados al mismo tiempo con otras drogas eficaces en esta parasitosis ?. Todos los pacientes fueron controlados clínicamente con el objeto de constatar posibles manifestaciones de intolerancia y apreciar variaciones de los síntomas aue presentaban al comienzo del tratamiento, especiaimente el prurito anal o nasal.

Interesados en evitar reinfecciones, se dió información sanitaria elemental a ambos grupos estudiados, con especial referencia a las medidas destinadas a interceptar el ciclo epidemiológico en esta baracitosis. tales como: aseo repetido de manos y uñas, antes de las comidas y después de defecar; uso de calzón apretado o cerrado en los tobillos para dormir; hervido de esta prenda de ropa todas las maŕэnas; uso de pomadas o vaselina sólida en la región anal durante la noche; aseo anogenital matutino; aseo con agua hirviendo de bacinicas o taza del W. C. Estas medidas higiénicas debieron ser cumplidas iambién por los componentes del grupo familiar que se encontraban libres de parásitos.

Finalmente, a los enfermos del Grupo II se les practicó estudio coprológico mediante tres exámenes de deposiciones, día por medio, según técnica de concentración, con el objeto de tener una medida indirecta de las condiciones de higiene ambiental de cada caso, las que son fielmente traducidas por los hallazgos del es iudio coprológicos.

\section{RESULTADOS}

Resultados del Grupo 1. En el tratamiento de los 23 niños de este grupo, se utilizaron dosis de Citrato de piperazina que fluctuaron entre 800 y 3.000 milígramos diarios, siendo las dosis totales entre 12 y 45 gramos por cura. El resultado de la terapéutica está consignado en la $\mathrm{Ta}-$ bla I, donde los pacienies han sido agrupados de acuerdo a los controles parasitológicos que se les practicaron y que han sido denominados "inmediatos" $y$ "alejados" según el menor o mayor tiempo transcurrido después de la cura. Se trató también de observar diferencias de resultados entre los nin̄os pre-escolares y escolares, grupos que difieren en cuanto a posibilidades de reinfección.

\section{TABIA I}

REGUTATADOS DEL TRATAMTENTO CON CITRATO DF PIPERATTNA EN 23 NINOS PARASTTATDOS FOR ENTFROBIDS TERMICULARIS. SEGLN GRUPOS DE TDAD $\mathbf{Y}$ CONTROL TRTALIZADO.

\begin{tabular}{|c|c|c|c|}
\hline Edad & $\begin{array}{c}\text { No de nifnos } \\
\text { tratados }\end{array}$ & $\begin{array}{c}\text { Negativos } \\
\text { en control } \\
\text { "Inmediato" }\end{array}$ & $\begin{array}{l}\text { Negativos } \\
\text { en control } \\
\text { "glejado" }\end{array}$ \\
\hline 1 a. 5 añas & 10 & 8 & 10 \\
\hline $\begin{array}{l}6 \text { afnos } \\
5 \text { mass }\end{array}$ & 13 & 12 & 13 \\
\hline Totales & 23 & $20(86 \%)$ & $23(100 \%)$ \\
\hline
\end{tabular}

LIama la atención al estudiar esta Tabla que en el control efectuado pocos días después del tratamiento, es decir, "inmediato", aparece un menor número de niños libres de parásitos $(86,9 \%)$ que en el "alejado", donde se logró la eliminación de este verme en el $100 \%$ del grupo tratado. No se advierte ninguna diferencia entre los grupos de edades ya que existió en ambos un $100 \%$ de curaciones.

En este grupo no existió dificultad para la ingestión del jarabe, que posee un agradable sabor. Por otra parte, las manifestaciones de intolexancia fueron extraordinariamente raras $y$ de escasa cuantia, puesto que sólo en tres casos existió dolor abdominal atribuible a la ingestión del medicamento $y$ en un caso se agregó además sensación de náuseas al finalizar la cura.

Resultados del Grupo II. Este grupo estuvo compuesto por 27 niños. Las dosis totales utilizadas fluctuaron entre $13 \mathrm{y}$ 52 gramos, en los niños que recibieron la dosis máxima total. Los componentes de este grupo también fueron estudiados bajo las mismas normas de con irol que el anterior, consignándose en la Tabla II los resultados de los controles "inmediatos" y "alejados" practicados después del tratamiento, diferenciándose dos grupos de edades. 
TABLA II

RESULTADOS DEL TRATABIENTO CON FOSFATC DE PIPERAZINA EN 27 PACIENTES PATASITA. DOS POR ENTEROBJUS PERWICULARIS, SITCIN GRDPOS DE EDAD Y CONTROL REALIZALO'

\begin{tabular}{|c|c|c|c|}
\hline $\begin{array}{c}\text { Eded } \\
\cdots\end{array}$ & $\begin{array}{l}\text { No de pa- } \\
\text { clentea } \\
\text { tratados }\end{array}$ & $\begin{array}{l}\text { Negatitros } \\
\text { en control } \\
\text { "Inmediate" }\end{array}$ & $\begin{array}{l}\text { Negatlvos } \\
\text { ep control } \\
\text { "alejado" }\end{array}$ \\
\hline 1 a 5 años & 13 & 13 & 13 \\
\hline 6 a 14 años & 14 & 10 & 12 \\
\hline Totsles & 27 & $23(85 \%)$ & $25\left(92 F_{(s)}\right)$ \\
\hline
\end{tabular}

Nuevamente se puede observar en esta Tabla que existe una menor nroporción de curaciones en el momento del control "inmediato" que en el "alejado", 85,9\% contra $92.6 \%$, respectivamente. Tampoco se advierten diferencias marcadas entre los grupos de edades estudiados.

En este grupo de pacientes tratados con Fosfato de piperazina no existió dificultad para la ingestión del medicamento, a pesar de venír baio la forma de tabletas, por cuanto en los niños menores fueron desleidas en agua edulcorada. Las manifestaciones colaterales dependientes del tratamiento fueron, en general, de relativa frecuencia e intensidad; las más constantes fueron las de orden alérgico tales como urticaria o prúrigos, que se observaron en 3 pacientes $\mathrm{y}$ las de aceleración del tránsito intestinal consignadas en igual número. Ningún paciente debió suspender el tratamiento por efectos colaterales del medicamento.

Resultados de la encuesta coprológica en el Grupo II. Se pudo observar que en $17(62,9 \%)$ de los 27 enfermos, el estudio coprológico reveló la presencja de otros enteroparásitos patógenos, siendo los más frecuentes Giardia lamblia en 12 casos y Entamoeba histolytica en 6 casos; además, un niño de 3 años 9 meses presentó ooquistes de Isospora belli, en sus excrementos. Estas cifras se consideran basiante elevadas en relación con las habituales en nuestro medio ${ }^{14}$, hecho que está confirmando la impresión primitiva de aue el grupo de pacientes estudiados provienen de un medio de alta contaminación parasitológica y de precarias condiciones socio-económicas.

\section{COMENTARIO}

No cabe duda que en el momento actual ias sales de dietilendiamina (Piperazina) constituyen la medicación de elección en el tratamiento de las infecciones por Enterobins vermicularis. Sin embargo, no esiá dicha la última palabra en cuanto a dosis del tratamiento se refiere. In tendencia a disminuir el número de días de medicación propuesta por algunos autores tales como Goeters ${ }^{2}$, Brown ${ }^{2}$ $\mathrm{y}$ otros ${ }^{1}$, parece no haber obtenido siem-pre resultados halagadores o concluyentes, tal vez por las características biológicas y epidemiológicas particulares de esta

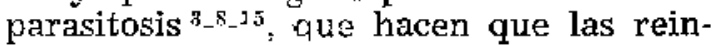
fecciones $y$ aún las retrofecciones sean frecuentes. Los trakaios realizados varinado la cuantía de las dosis diarias prescritas, tampoco son demostrativos en cuanto a obtener éxitos proporcionales a ellas y es así como utilizando dosis entre 50 y 100 milígremos nor kilógramo de peso corporal y por día, diferentes auto. res publican porcentajes de éxitos más o menos semejantes "12_16.

Los resuliados de nuestras dos series de pacientes estudiados, debemos analizarlos por separado. Previamente creemos de interés dejar establecido que de acuerdo a lo observado en las tablas de resultados, consideraremos como totalmente curados a los pacientes cuyos controles "alejados" fueron negativos, por cuanto en ambos grupos de ensayo se observó persistencia de h!zevos de oxyurus en exámenes realizados a poco de finalizada la cura, tratándose probablemente de huevos residuales en las márgenes del año, o en las deposiciones de estos mismos individuos. Es de interés señalar además, oue en 11 pacientes del Grupo I a los que fué posible practicarles una tercera serie de control sogún la técnica de Graham entre 2 y 6 meses después del tratamiento, persistian aún negativos, tal como en el control que hemos dado en llamar "alejado". Nos parece de interés insistir en las características del criterio de curación aplicado en ambas series presentadas en este trabajo, por cuanto en la mayoría de las publicaciones revisadas ${ }^{5} 12_{2} 1 n$ el control de la terapia es realizado pocos días después de finalizada ésta, factor que probablemente altere los resultados obtenidos y haga 
menospreciar las dosis o esquemas de tratamienio empleados. Deducimos de nuestra casuística aue el control parásitológico en esta enfermedad debe ser realizado alrededor de $\mathbf{1 5}$ días después de finalizada la cura y mediante el estudio de un número suficiente de placas de papel celofán engomado, que estimamos entre 5 y 10 , tomadas en días consecutivos.

Los resultados obtenidos con el empleo del Cirrato de piperazina en nuestra serie son bastante halagadores y sobrepasan a los mencionados en otras publicaciones ${ }^{5} 12_{-} t *$, en que se ha trabajado con métodos comparables. También el Fosfato de piperazina reveló poseer estimables condiciones como droga antioxyuricida, y la proporción de éxitos obtenida se eneuentra al mismo nivel, o mejor aún, que las alcanzadas con otras sales de la piperazina ${ }^{2}{ }^{6}{ }^{12}$. La diferencia que se aprecia entre los porcentaies de mejorías conseguidos con el uso de estas dos sales de la piperazina, a nuestro parecer no son concluyentes $y$ poeden ser explicadas por variaciones en el tamaño de las muestras estudiadas, por diferencias en el número de placas de control, o quizás por mecanismos dependientes de la tolerancia observada para ambas drogas, la que en la literatura está descrita habitualmente como buena 1_2_12. En el Grupo I tratado con Citrato de piperazina sólo 3 niños $(13,0 \%)$ de 23 revelaron ciertas alteraciones dependientes de la droga y siempre fueron de escasa intensidad; del mismo modo, en el Grupo IT tratados con Fosfato de piperazina $\overline{4}(14,8 \%)$ de 27 pacientes tuvieron síntomas secundarios y siempre de escasa cuantía.

Los fenómenos de reinfección no pueden ser invocados para explicar estas diferencias, por cuanto ambos grupos en estudio son comparables en sus características socio-económicas $\mathrm{y}$ de higiene personal y ambiental, aún cuando el fracaso terapéutico en dos niños del Gruno II se debió, a nuestro juicio, a incumplimiento de las normas higiénicas recomendadas. Por otra parte, debemos reconocer aue no siempre las indicaciones en esie sentido, proporcionadas junto con la administración del medicamento, se cumplen en forma estricta aún cuando se trata de normas tan elementales que pueden ser aplicadas con relativa facilidad y algunas de las cuales constituyen nociones mínimas de la higiene personal corriente.

En el Grupo II de pacientes, tratados con Fosfato de piperazina, se estudió también el efecio de la mejoría parasitológica en la sintomatología que preseritaban los enfermos antes del tratamiento. Se consideró como síntoma fundamental el prurito, tanto anal como nasal, y se pudo apreciar que prácticamente en todos los casos existió evidente desaparición o disminución de las molestias, aún en aquellos casos en que no se logró la erradicación del parásito. Este hecho nos permite manífestar que está plenamente justificado el tratamiento de individuos aislados en aquellos casos en que la intensidad de las molestias lo exigen y existe la imposibilidad de efectuar tratamientos de carácter familiar.

Finalmenie debemos establecer que los resultados obtenidos en nuestra serie con el empleo del Citrato y del Fosfato de piperazina en las infecciones por Enterobius vermicularis, durante períodos prolongados, son comparables o superiores a los publicados por otros autores $y$ al parecer independientes de las medidas higiénicas recomendadas, de la edad de los pacientes y de sus probabilidades de reinfección.

\section{RESUMEN}

Se trataron con diferentes sales de piperazina, dos grupos de enfermos parasitados por Enterobius vermicularis, que en total comprendió a $\mathbf{5 0}$ individuos.

El Grupo I, compuesto por 23 nin̄os, entre 1 y 11 años de edad, fué tratado con jarabe de Citrato de piperazina en dosis de 80 milígramos por kilógramo de peso, diario, durante 15 días. El Grupo II formado por 27 niños, entre 1 y 14 años, fué tratado con tabletas de Fosfato de piperazina en dosis y días similares al grupo anterior.

Jun co con la prescripción del medicamento se indicaron medidas de higiene elementales. El control de Ia terapia fué realizado mediante la técnica del papel celofán engomado aplicado en series de 5 placas en días sucesivos en el Grupo I y de 10 placas en igual forma en el Grupo II. 
Los resultados "alejados" del tratamiento revelaron mejoría en todos los niños del Grupo I y en 25 de $27(92,6 \%)$ pacientes del Grupo II.

La administración de las drogas en estudio provocaxon ciertas manifestaciones de intolerancia, las que fueron más frecuentes en el grupo tratado con Fosfato de Piperazina, siendo las más importan. tes las de carácter alérgico y las dependientes del aparato digestivo.

\section{SUMMARY}

\section{OXYURIASIS IN CHILDHOOD}

Two gruops of sick children infected with ENTEROBIUS vERMICULARIS, totalizing 50 individuals, were treated with different salts of piperazine.

The group I composed of 23 children aged from 1 to 11 years, was treated with syrup of piperazine citrate in daily doses of $80 \mathrm{mg}$. per $\mathrm{kg}$. body weight during 15 days. The group II formed by 27 children aged from 1 to 14 years, was treated with tablets of piperazine phosphate in doses and for days similar to the former group.

Together with the prescription of the medicament, elementary hygienic measur. es were indicated. The outcome of the therapy was controlled by means of the iechnique of adhesive cellophane paper which was applied to the group I in series of 5 strips on successive days and to the group II in series of 10 sirips in the same way.

The late results of the treatment revealed an improvement in all the children of the group $I$ and in 25 of the 27 patien's $(92,6 \%)$ of the group II.

The administration of the drugs we are studyin, provoaued certain manifestations of intolerance which were more frequent in the group treated with piperazine phophate; the most importan $i$ ones were those of alleroic character and those depending of the digestive system.

\section{ZUSAMMENFASSUNG}

\section{OXYURIASIS IM KINDESACTER}

Zwei Gruppen von Kranken mit ENTEROBIUS verMICULARIS-Befall, insgesamt 50 Personen, wurden mit verschiedenen $\mathrm{Pi}$ perazin-Salzen behandelt.

Die aus 25 Kindern im Alter von 1 bis 11 Jahren bestehende Gruppe I wurde vierzehn Tage lang mit Piperazincitrat-
Sirup in Dosen von $80 \mathrm{mg}$. per kg. Körpergewicht behandelt. Die von Kindern im Alter von 1 bis 14 Jahren gebildete Gruppe II wurde mit Piperazinphosphat-Tabletten in gleichen Dosen und so lange wie die andere Gruppe behandeIt.

Gleichzeitig mit dem Verschreiben des Medikaments wurden elementare hygienische Massnahmen angeordnet. Der Behandlungserfolg wurde mit der Technik des Cellophanklebepapiers kontrolliert, das an aufeinanderfolgenden Tagen in der Gruppe I in Serien von 5 Streifen und in der Gruppe II in Serien von 10 Streifen appliziert wurde.

Die Spätresultate der Behandlung ergaben eine Besserung bei allen Kindern der Gruppe I und bei 25 von 27 Patienten $(92.6 \%)$ der Gruppe II.

Die Verabfolgung der der Prüfung unterliegenden Arzneimittel verursachte gewisse Intoleranz-Erscheinungen, von denen die wichtigsten diejenigen allergischen Charakters und die auf den Verdauungesapparat bezuglichen waren.

\section{BIBLIOGRAFIA}

1.-ATTAB, A. y RAMTREZ, M. - Oxyuriksis, ensayo terapéutleo con hexentirato ae piperazina en cuatro dia de tratamiento. Bol. Chlleno. Parast.. 14:23, 1059

2.-BROWN, B. W.; EAM-FATN, Ch. Y HUSSEY, T. Treatrant of Enteroblasls and Ascaridiasis with Piperazine. J,A.M.A, 161:515, 1956.

3.-CHANDLFR, A. - Introduction to Parasitologs. 99 bult., Wileg and Sons. New York, 1954.

4.-DONOSO, F, - Infectones por Ascarts limbricoldes en nifios tratados con Citrato de Piperazlma. Eol, chlleno Parasit., 10:49. 1955.

5.-FAIGUENBAUM, J.: GONZALEZ, G.; PENA. J. Y MAFFIOIETTI, F. - Tratamiento de la oxvuriasis con Dletilendlamins (Plperazlna). Bol. Chllevo, Paraslt., $11: 51,1956$.

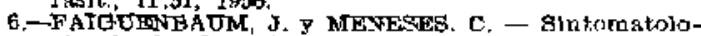
gla do la Oxyuriasis, Bol, Chlleno Paraste, 9:10, 1954.

7-FANTA, $\mathbf{E}$ - Princhajes tratamlentos de las parasitosis intestimajes en los nifios. Bol. Chileno Infitosis intestinajes en los nilpos.

B.-FAUST, E. O. - Finm Helmintology, 79 Ed. Lea and Frbiser. Philabeiphia, 1949

9.-GOFYLRS, w. y NORDBECK, 8 - Zur Bebandlung der Oxviring im Kintersatict: Weltere Fufahungen mit Uvilon. Medizinische (Sttutgart), 41:1449. 1955.

10. GRABAAM, C. F. - A devtee for the dlagnosis of Fnterofilis infoction. Am. J. TTop. Med. 21:159, 1941.

11.-HEWITT, R. I; WALLACE, W. \$; WHITE, E. y SUBARROW, G. - Expertmental chemotherapy of fliariasis: experimental methorss for testing drues against neturally acquired fljarlal intectjons in cottan rats and dosts. J. Lab. Clin. Med., 32:1293. 1947.

12,-MOJRIQUAND. G; ROMAN, $\mathbf{B}$, y COISNARD, J. Exset de traitoment de I'oxyarose par la plperazine. $J$, de Med. de Lyon, 32:189, 1951.

13.-MENESEs, C. - Contribución al estudia clinicoeptdemolóngico de It Oxyurlasis en Chlle. Tesis, 1952.

14.-NEXTHME, A.; SILVA, R. y SOTOMAYOR. R. Futeroperastiota entre escolares de la provincla de Santiago. Bol. Chlleno Paragit., 9:70. 1954.

15.-PESSOA, \$. B. - Parasitología Métca. 53 Eutcao. Livfaria Editorg Guanabara, Rio de Janetro, 1958 .

16.-WHTIE, H. R. g STANDEY, O. D. -Piperazine in the trentment or theoristoms in chsidren. Repor: on a cilnfeal trial. Brit. Med. J., 2:755, 1983 . 